\title{
Multi hop and Multi Direction Selection Data Dissemination Technique for Road Safety in VANET
}

\author{
Manpreet Kaur ${ }^{1}$, Navjot Bhullar ${ }^{2}$ \\ Sri Sai College of Engineering \& Technology, Manawala ${ }^{1,2}$
}

\begin{abstract}
There is a major increase within the rates of car accidents in countries round the world and additionally the casualties concerned ever year. New technologies are explored about the Vehicular ad hoc Network (VANET) as a result of the rise in transport traffic/congestions around us. Vehicular communication is extremely vital as technology has evolved. The analysis of VANET and development of proposed systems and implementation would increase safety among road users and improve the comfort for the corresponding passengers, drivers and also other road users, and an excellent improvement within the traffic efficiency would be achieved. the security problems are described and improved with the proposed technique.
\end{abstract}

Index Terms: Vehicular ad hoc networks (VANETs), next available node, MHMD AMD.

\section{INTRODUCTION}

Vehicular Ad hoc Network (VANET) may be a kind of Mobile Ad hoc Network (MANET), where mobile nodes are vehicles [5]. VANETs offer wireless communication between vehicles and road facet equipments [1]. Vehicles that are in radio range of every alternative will communicate with each other. In VANETs, delivery isn't single hop rather multi hop delivery is finished and even the vehicle that is miles far from destination also can send their request like- traffic conditions, obstacle information may be obtained by the vehicles after they are currently not in the city [3]. VANETs are distributed, self-organized and potentially highly mobile networks of vehicles interacting via wireless media [2].

In vehicular ad hoc networks, there's very high mobility in which every vehicle node act as a router as well as host and sending packets to alternative mobile nodes and changing their topology very fast [3]. In vehicular ad hoc networks topology keeps on changing and also vehicles aren't always connected to the network. There are frequent disconnections in VANETs. Therefore, routing protocols used in MANETs aren't essentially be suitable for VANETs as in MANET's protocols there's an implicit assumption of network connectivity [2]. Intermittent connectivity, frequent changes in network topology and low reception rate are those properties that distinguish VANETs from alternative kinds of ad hoc networks [2]. Routing will be defined as finding optimal path between source and destination node and then sending message on that path so that message will reach its destination easily, quickly and on time. The most drawbacks that need to be solved in VANETs are how to exchange information in scalable fashion [4]. The answer lies in data Dissemination Protocols. Data Dissemination Protocols differ from each other in terms of that some of them are employed in highway whereas others are used in urban areas and some can be employed in each scenarios. As in case of urban areas there are several number of intersection points and in large cities buildings will block communication among near vehicles though they're in transmission range of each alternative. So, it becomes necessary to forward information to those vehicles or Road side Units (RSU) that are near intersections so information packets mustn't be lost because of obstacles like buildings. Therefore, Protocols that are used for disseminating data in urban areas could differ from those who are used for propagating information on highways as highways shows one dimensional topology.

\section{Challenges in data Dissemination:}

Data dissemination may be a process of spreading data or information over distributed networks [1]. So, data dissemination in VANETs improves the efficiency of traffic system. It also improves the quality of driving. Though this process looks to be very easy however in reality it's robust for vehicles to communicate among themselves because of large number of vehicles on road. So, it becomes very challenging task for vehicles to transmit information over the network [7]. Some of the most issues during dissemination of data are:

\section{i) High mobility and Frequent Disconnections:}

The big challenge in VANETs is the high mobility and frequently disconnected topology at totally different regions of the city. The traffic density is low throughout the night and in suburban areas, but network node density is very high in urban areas and especially throughout the peak hours in as CH. In [6] [15], the authors consider local mobility to day time, which causes frequently network disconnection. 
There is no easy 'one-for-all' solution for disseminate data to all recipients spreading across the city [1].

ii) Data Transmission in presence of Disconnection:

The second main challenge in VANETs how to disseminate data over the network with less delay and before occurring the disconnections among vehicles. When target vehicle moves closer to the roadside unit and placed in densely area, disconnection is less concern. However the major drawback is when different vehicles those are in radio range of one another requesting a similar data at the same time and sharing the wireless media then utilization of bandwidth is the key issue. when a vehicle reaches among the one-hop range of the road side units, data may be transmitted to the vehicle at the highest throughput. Thus a vehicle passes by the roadside unit, it's most desirable to extend the connection time between the vehicle and road side unit so as to spread more data [1].

iii) Data Distribute over the Mesh Nodes:

For efficient data dissemination, many roadside units are connected together to make an infrastructure like mesh network and cooperatively disseminate data to the vehicles. So, it becomes very difficult how to distribute data within the mesh network [1].

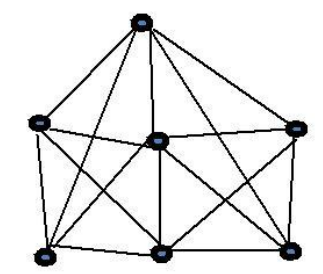

Figure1: Mesh Network

\section{Types of information Dissemination}

Data Dissemination may be a process of spreading data or information over distributed wireless networks. Aim of data dissemination is the optimum use of network resources to serve the data desires of all users [1]. Differing kinds of data dissemination employed in VANETs are:

a. $\quad \mathrm{V} 2 \mathrm{I} / \mathrm{I} 2 \mathrm{~V}$ Dissemination (Vehicle to infrastructure or RSU)

b. V2V Dissemination (Vehicle to vehicle)

c. Opportunistic Dissemination

d. Peer-to-peer Dissemination

e. Cluster based Dissemination

\section{RELATED WORK}

Harjivan Preet Kaur et al. [8] [2017] Data dissemination in VANET may be a puzzling task. Various protocols and techniques are used for data dissemination. For this, it's essential to route information efficiently from source to destination in order to avoid accidents or collisions. This paper highlights different data dissemination techniques in a clustered and unclustered environment in VANET.

Er. Gaganpreet Kaur et al. [9] [2016] Vehicular plays a very important role in everyday world within the field of automobiles. VANETs are the subclass of mobile ad-hoc Networks that have no central infrastructure. Nodes are highly mobile in VANET. VANET is distinguished from Manet by their features, design and applications. A vehicular AdHoc Network or VANET may be a sub kind of Mobile Ad-Hoc Network or Manet that gives communication between vehicles and between vehicles and road-side base stations with an aim of providing efficient and safe transportation. A vehicle in VANET is considered to be an intelligent mobile node capable of communicating with its neighbours and alternative vehicles within the network. VANET introduces more challenges aspects as compare to Manet because of high mobility of nodes and fast topology changes in VANET. VANET basics structure, its architecture, challenges, applications are discussed. Data dissemination may be a challenging task as a result of by utilizing restricted bandwidth, maximum data needs to publicize over the conveyance network. Several researchers have provided many techniques to publicize data so that the data may be accessed more efficiently. Major stress is given to this topic where data dissemination types and its protocols are compared.

Maryam M. Alotaibi et al. [10] [2015] Distance may be a key measure when implementing timer-based dissemination protocols in vehicular Ad-hoc Networks (VANets). The transmission is deferred proportional to distance, aiming to order vehicles transmission, such that the farthest vehicle gets the highest opportunity to relay the message. This will ensure long hops on the road to speed up the dissemination and cover more nodes. However, in case of heterogeneous transmission ranges, the farthest distance will not ensure a proper choice of relay nodes to disseminate the message. Vehicles, whose transmission area enclosed by the sender or have small non-covered transmission area, may be chosen as relay vehicle. This could inhabit alternative nodes from relaying the message and finish the dissemination process early and before it reach the desired region. The area Defer Transmission (ADT) dissemination algorithm. ADT enables 
every vehicle to independently decide to transmit or suppress transmission considering heterogeneous transmission ranges and the amount of area that may be covered by potential new transmission.

The performance of the proposed ADT algorithm has been evaluated using an actual road map with complex road scenarios and real movement traces. It's also been thoroughly investigated and compared with alternative distancebased algorithms. The results demonstrate that ADT achieves high delivery ratio, high propagation speed and fewer relay ratio with fewer hops that reach long distances.

\section{PROPOSED METHOD}

The data dissemination schemes the algorithm assumed that each node within the network is totally equipped with GPS, i.e. every vehicle is aware of the precise geographical location of the opposite vehicles that are within the communication vary. Frequent change of data ought to occur at nearer intervals. Relative speed between vehicles ought to amendment slowly so as to possess longer updating interval. Whenever an accident takes place, the accident vehicle broadcasts an alert message to all the other vehicles within its communication range. So all the vehicles within the communication range of the accident vehicle will be receiving the alert message, after receiving the message it will not rebroadcast it immediately. It will wait for some time and will perform MHMD algorithm the last vehicle in the communication range will be considered. The MHMD counter based algorithms takes the help of network usage into account in order to forward the packets to other nodes, while in probability based method, regardless of network status it makes a probabilistic choice on packet forwarding to the nodes. In the counter based method, each node has been set a timer for each non duplicate message it receives. Each time when a node receives non duplicate message, delay time for each timer is set randomly and decremented afterwards Whenever a node overhears the duplicate messages from its neighbors, the counter gets increased. If the counter exceeds the threshold (Max-Count) when the timer expires, then the node discards the packet and suppresses the forwarding in order to prevent the repetition of packets. Thus counter based method is more robust in various network wide broadcasting scenarios due to its adaptive ability in controlling the probability based packet forwarding in conjunction with the node density

ALOGRITM1: Multi Hop Multi Direction Transmission

$1:$ for time $=1$ to simulation time

2: for $\mathrm{i}=1: \mathrm{N}$, where $\mathrm{N}$ the number of nodes that located in the network

3: for nodeid $=1$ :nodeid node id number

within the network

4 Find neighbour ant update count

5: if location (i) within the loc(nnnodeid);

6: Add i to (updated count); calculate updated count with time and direction

7:else

8:update routing table and broadcast successfully

9: endif

10: end

11: end

12: end

\section{RESULTS AND DISCUSSIONS}

\begin{tabular}{|l|l|l|}
\hline S. No. & Parameter & Value(s) \\
\hline 1 & Simulator used & NS 2.35 \\
\hline 2 & Simulation Time & 10 Secs \\
\hline 3 & Simulation Area & 500 X 500 \\
\hline 4 & MAC & 802.11 \\
\hline 5 & Number of nodes & 60 \\
\hline 6 & Speed of Nodes & 2 to $16(\mathrm{~m} / \mathrm{sec})$ \\
\hline 7 & Mobility Model & Random Waypoint \\
\hline
\end{tabular}

The performance metrics used for evaluating the performance of the proposed clustering algorithm are as follow:

Communication delay:It is common in VANET that the nodes move within a certain transmission range. Therefore, network performance directly depends on the number of hops with average delay in a vanet. Delay is one of the key parameter to be considered for vehicular network traffic. It is defined as the time taken for a packet to be transmitted across a network from source to destination. The analysis of delay between AMD and MHMD are shown in fig. 5 that shows the communication delay using MHMD is very low as compared to AMD, but due to increase in vehicle density the communication delay increase in both the cases but in our proposed technique the results are much better. 


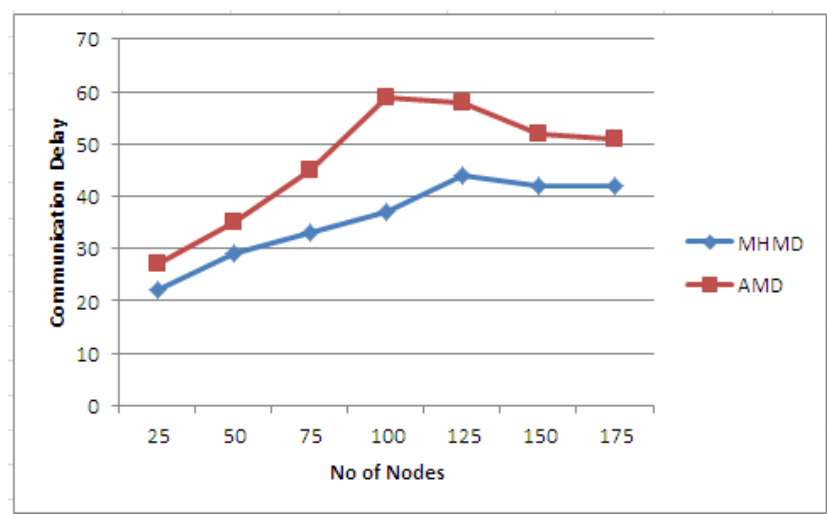

Fig.1. Communication Delay

\begin{tabular}{|l|l|l|}
\hline & \multicolumn{2}{|c|}{ Communication Delay } \\
\hline No of Nodes & AMD(Existing) & MHMD(proposed) \\
\hline 25 & 27 & 22 \\
\hline 50 & 35 & 29 \\
\hline 75 & 45 & 33 \\
\hline 100 & 59 & 37 \\
\hline 125 & 58 & 44 \\
\hline 150 & 52 & 42 \\
\hline 175 & 51 & 42 \\
\hline
\end{tabular}

\section{Delivery ratio}

Packet delivery ratio is the ratio of the number of messages received by the destination to the number sent by the sender.[34] The analysis of Delivery ratio between AMD and MHMD are shown in fig.6 that shows the Delivery ratio using MHMD is very high as compared to AMD but due to increase in vehicle density the delivery ratio increases in both the cases but in our proposed technique the results are much better. high as compared to AMD but due to increase in vehicle density the delivery ratio increases in both the cases but in our proposed technique the resul ts are much better

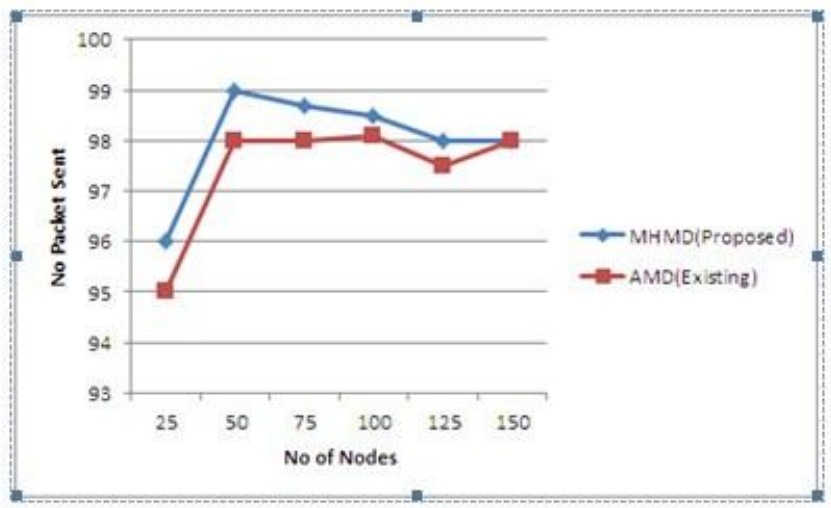

Fig2 Delivery ratio

Table 2 Delivery ratio

\begin{tabular}{|l|l|l|}
\hline & Delivery ratio \\
\hline No Vehicles & AMD(Existing) & MHMD(Proposed) \\
\hline 25 & 95 & 96 \\
\hline 50 & 98 & 99 \\
\hline 75 & 98 & 98.7 \\
\hline 100 & 98.1 & 98.5 \\
\hline 125 & 97.5 & 98 \\
\hline 150 & 98 & 98 \\
\hline 175 & 96.5 & 97 \\
\hline
\end{tabular}




\section{Simulation time}

The analysis of Data loss between AMD and MHMD are shown in fig.7 that shows the MHMD loss using MHMD is low as compared to AMD.

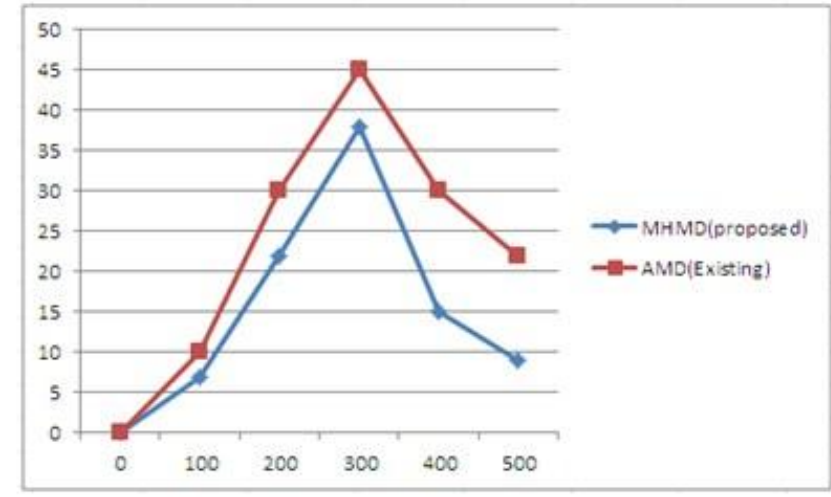

\begin{tabular}{|l|l|l|}
\hline & Data Loss \\
\hline Simulation Time & EBRS(Existing) & DTA(proposed) \\
\hline 0 & 0 & 0 \\
\hline 100 & 10 & 7 \\
\hline 200 & 30 & 22 \\
\hline 300 & 45 & 38 \\
\hline 400 & 30 & 15 \\
\hline 500 & 22 & 9 \\
\hline
\end{tabular}

\section{CONCLUSION}

VANETs improve highway safety. However, researchers have pointed out that VANETs are facing a number of security threats, which might impair the efficiency of VANETs and even life safety. One of these threats is Data loss $\mathrm{s}$, in which a malicious vehicle creates illusion of traffic congestion by claiming multiple identities. Not only does this create an illusion, it has the potential to inject false information into the networks via a number of fabricated nonexisting vehicles. We propose time stamp approach to detect the Data loss. It maintains a unique identity of each node. The proposed technique identifies Data loss from the network and provides efficient transmission in the network as compared to previous technique

\section{REFERENCES}

[1] Mor Annu, "Study of Different Type of Data Dissemination Strategy in VANET", International Journal of Engineering Science and Innovative Technology (IJEST), Vol.1, pp. 6-8, nov-2012

[2] Daraghmi Yousef-Awwad, Yi Chih-Wei, Stojmenovic Ivan, Abdulaziz King, "Forwarding Methods in Data Dissemination and Routing Protocols for Vehicular Ad Hoc Network,” pp. 74-79, IEEE 2013.

[3] Dubey Brij Bihari, Chauhan Naveen, Kumar Prashant, “A Survey on Data Dissemination Techniques used in VANETs," International Journal of Computer Applications, vol.10, pp. 5-10, nov-2010.

[4] Nadeem Tameer, Shankar Pravin, Iftode Liviu, "A Comparative Study of Data Dissemination Models for VANETs", IEEE july-2006.

[5] Kakkasageri M.S and Manvi S.S, "Regression based critical information aggregation and dissemination in VANETs: A cognitive agent approach", july 2014.

[6] Tomar Pratibha, Chaurasia Brijesh Kumar, Tomar G.S., "State of the Art of Data Dissemination in VANETs," International Journal of Computer Theory and Engineering, Vol.2, No.6, pp. 957-962, Dec-2010.

[7] Sushil Kumar, Sudesh Rani, "A Study and Comparative Analysis of Cluster-based data

[8] Harjivan Preet Kaur and Amarpreet Singh, " Review on Data Dissemination Techniques in VANET", International Journal of Innovations in Engineering and Technology (IJIET), Vol.8 , , ISSN: 2319 - 1058, pp. 205-208., 2017.

[9] Er. Gaganpreet Kaur and Dr. Sandeep Singh Kang, " Study of Various Data Dissemination Types and its Protocols - A Review", International Journal of Advanced Research in Computer Science, Vol.7, ISSN: 0976-5697, pp.133, 2016.

[10] Maryam M. Alotaibi and Hussein T. Mouftah, " Data Dissemination for Heterogeneous Transmission Ranges in Vanets", IEEE Conference on Local Computer Networks, pp.818-825, 2015. 